Check for updates

Kent

Cite this as: $B M J 2022 ; 376: 0411$ http://dx.doi.org/10.1136/bmj.0411 Published: 16 February 2022

\title{
Covid-19: Vaccine will be offered to 5-11 year olds throughout UK
}

\section{Jacqui Wise}

England and Northern Ireland have followed Scotland and Wales in announcing that all children aged 5-11 years will be offered the covid-19 vaccine, in line with advice from the Joint Committee on Vaccination and Immunisation (JCVI).

England's health and social care secretary, Sajid Javid, said that the offer was "non-urgent" and would be made to all children in April.

The JCVI's advice was handed to the UK government more than a week ago and was expected to be published on 11 February. Media reports have suggested that a disagreement with the government led to the delay in publication. ${ }^{2}$

Overall, the committee agreed that the potential health benefits of vaccination were greater than the potential health risks. Children will be offered two $10 \mu \mathrm{g}$ doses of the Pfizer-BioNTech vaccine with an interval of at least 12 weeks between doses.

The advisory body said that the offer of covid-19 vaccination should not displace the delivery of other immunisation programmes.

Over $85 \%$ of all children aged 5-11 will have had prior SARS-CoV-2 infection by the end of January 2022, with roughly half of these infections due to the omicron variant, the JCVI said. Natural immunity arising from prior infection will contribute towards protection against future infection and severe disease, it advised.

The committee said that, in comparison with the rest of the population, children aged 5-11 had the very lowest risk from covid-19. However, vaccination of this group is anticipated to prevent a small number of hospital and intensive care admissions and would provide short term protection against non-severe infection. However, the JCVI says that the extent of these effects is highly uncertain and is closely related to the timing, size, and severity of any future waves of infection. It said that it could not determine the impact of vaccination on school absences.

Vaccination of children aged 5-11 who are not in a clinical risk group is not expected to have an effect on the current wave of omicron infection. The potential benefits of vaccination will apply mainly to a future wave of infection.

\section{Non-urgent offer}

On 15 February Wales was the first country in the UK to announce that it would offer the vaccine to children aged 5-11. The following day Nicola Sturgeon, Scotland's first minister, confirmed that health boards would deliver the vaccine to children in Scotland.

At that point the Department of Health and Social Care was still saying that it was "reviewing the JCVI's advice as part of wider decision-making ahead of the publication of our long-term strategy for living with covid-19. More detail will be set out shortly."

However, later on 16 February Javid said, "I have accepted the advice from the independent Joint Committee on Vaccination and Immunisation to make a non-urgent offer of covid-19 vaccines to all children aged 5-11 in England."

He added, "Children without underlying health conditions are at low risk of serious illness from covid-19, and the priority remains for the NHS to offer vaccines and boosters to adults and vulnerable young people, as well as to catch up with other childhood immunisation programmes.

“The NHS will prepare to extend this non-urgent offer to all children during April so parents can, if they want, take up the offer to increase protection against potential future waves of covid-19 as we learn to live with this virus."

On 22 December last year the Medicines and Healthcare Products Regulatory Agency approved the Pfizer covid-19 vaccine for children aged 5-11. At the end of January the NHS in all four UK nations then started to offer the Pfizer vaccine to children aged 5-11 who had specific medical conditions and those who lived with immunosuppressed people. The vaccine has also been administered to millions of children in this age group in other countries including Germany and the US.

Adam Finn, a JCVI member and professor of paediatrics at the University of Bristol, commented, "The risks posed by covid in this age group are low, but they exist, and parents may wish to reduce them by having their children immunised. The programme will not impact significantly on the current omicron wave in children but may reduce the risks children may face in a possible future wave of this or another variant.”

Another JCVI member, Matt Keeling, professor of populations and disease at Warwick University, said, "It's great to see this statement made public, and I'm sure it will be a substantial relief to many parents. We now have a very good understanding of the risks from the mRNA vaccines, and therefore know that the risks to this age group-who receive a smaller dose-are minimal. However, given that omicron is now in decline and many children will already be protected through infection, the short term benefits are also likely to be small."

Welcoming the announcement, Brian Ferguson, associate professor of immunology at the University of Cambridge, said, "There is an argument that it is now too late to offer the vaccine to this age group, as covid has torn through primary schools this winter; however, there are children who have not yet been exposed to covid who will benefit from immunisation, 
and immunological data indicates that vaccination following infection generates powerful, broadly neutralising antibodies that protect from most or all tested SARS-CoV-2 variants.

"Covid vaccination can also in some cases help reduce long covid symptoms.”

Update: On 17 February 2022 we added several paragraphs to this article to include Northern Ireland and to link to the JCVI's statement and comments.

1 Department of Health and Social Care. Independent report: JCVI statement on vaccination of children aged 5 to 11 years old. 16 Feb 2022. https://www.gov.uk/government/publications/jcviupdate-on-advice-for-covid-19-vaccination-of-children-aged-5-to-11/jcvi-statement-on-vaccinationof-children-aged-5-to-11-years-old

2 Davis N, Mason R. Plans to delay Covid jabs for UK children aged five to 11 criticised. Guardian 2022 Feb 14. https://www.theguardian.com/society/2022/feb/14/plans-to-delay-covid-jabs-foruk-children-aged-five-to-11-criticised

This article is made freely available for personal use in accordance with BMI's website terms and conditions for the duration of the covid-19 pandemic or until otherwise determined by BMJ. You may download and print the article for any lawful, non-commercial purpose (including text and data mining) provided that all copyright notices and trade marks are retained. 